\title{
Decompressive Craniectomy Is Associated With Good Quality of Life Up to 10 Years After Rehabilitation From Traumatic Brain Injury
}

\author{
Rauen, Katrin ; Reichelt, Lara ; Probst, Philipp ; Schäpers, Barbara ; Müller, Friedemann ; Jahn, Klaus
} ; Plesnila, Nikolaus

\begin{abstract}
OBJECTIVES Traumatic brain injury is the number one cause of death in children and young adults and has become increasingly prevalent in the elderly. Decompressive craniectomy prevents intracranial hypertension but does not clearly improve physical outcome 6 months after traumatic brain injury. However, it has not been analyzed if decompressive craniectomy affects traumatic brain injury patients' quality of life in the long term. DESIGN Therefore, we conducted a cross-sectional study assessing health-related quality of life in traumatic brain injury patients with or without decompressive craniectomy up to 10 years after injury. SETTING Former critical care patients. PATIENTS Chronic traumatic brain injury patients having not $(\mathrm{n}=37)$ or having received $(\mathrm{n}=98)$ decompressive craniectomy during the acute treatment. MEASUREMENTS AND MAIN RESULTS The Quality of Life after Brain Injury questionnaire was used as outcome measure with a total score from zero to 100, representing lowest and best health-related quality of life, respectively. Health-related quality of life was compared between patients with or without decompressive craniectomy for the entire cohort, for the traumatic brain injury severity (mild, moderate, severe) measured by the initial Glasgow Coma Scale, for age and time variables (age at traumatic brain injury, age at survey, elapsed time since traumatic brain injury) using the Mann-Whitney U test. Differences were considered significant at a $\mathrm{p}$ value of less than 0.05.Decompressive craniectomy was necessary in all initial traumatic brain injury severity groups. Eight percent more decompressive craniectomy patients reported good health-related quality of life with a Quality of Life after Brain Injury total score greater than or equal to 60 compared with the no decompressive craniectomy patients up to 10 years after traumatic brain injury $(\mathrm{p}=0.004)$. Initially, mild classified traumatic brain injury patients had a median Quality of Life after Brain Injury total score of 83 (decompressive craniectomy) versus 62 (no decompressive craniectomy) ( $\mathrm{p}=0.028$ ). Health-related quality of life regarding physical status was better in decompressive craniectomy patients $(\mathrm{p}=0.025)$. Decompressive craniectomy showed a trend toward better health-related quality of life in the 61-85-year-old reflected by median Quality of Life after Brain Injury total scores of 62 (no decompressive craniectomy) versus 79 (decompressive craniectomy) (p $=0.06)$. CONCLUSIONS Our results suggest that decompressive craniectomy is associated with good health-related quality of life up to 10 years after traumatic brain injury. Thus, decompressive craniectomy may have an underestimated therapeutic potential after traumatic brain injury.
\end{abstract}

DOI: https://doi.org/10.1097/CCM.0000000000004387

Posted at the Zurich Open Repository and Archive, University of Zurich

ZORA URL: https://doi.org/10.5167/uzh-188712

Journal Article

Published Version 
Originally published at:

Rauen, Katrin; Reichelt, Lara; Probst, Philipp; Schäpers, Barbara; Müller, Friedemann; Jahn, Klaus; Plesnila, Nikolaus (2020). Decompressive Craniectomy Is Associated With Good Quality of Life Up to 10 Years After Rehabilitation From Traumatic Brain Injury. Critical Care Medicine, 48(8):1157-1164. DOI: https://doi.org/10.1097/CCM.0000000000004387 


\title{
Decompressive Craniectomy Is Associated With Good Quality of Life Up to 10 Years After Rehabilitation From Traumatic Brain Injury
}

\author{
Katrin Rauen, $\mathrm{MD}^{1-4}$; Lara Reichelt, $\mathrm{MD}^{1,2}$; Philipp Probst, $\mathrm{PhD}^{5}$; Barbara Schäpers ${ }^{1}$; \\ Friedemann Müller, $\mathrm{MD}^{1}$; Klaus Jahn, $\mathrm{PhD}^{1,6}$; Nikolaus Plesnila, $\mathrm{PhD}^{2,7}$
}

Objectives: Traumatic brain injury is the number one cause of death in children and young adults and has become increasingly prevalent in the elderly. Decompressive craniectomy prevents intracranial hypertension but does not clearly improve physical outcome 6 months after traumatic brain injury. However, it has not been analyzed if decompressive craniectomy affects traumatic brain injury patients' quality of life in the long term.

Design: Therefore, we conducted a cross-sectional study assessing health-related quality of life in traumatic brain injury patients with or without decompressive craniectomy up to 10 years after injury.

Setting: Former critical care patients.

Patients: Chronic traumatic brain injury patients having not ( $n=$ 37 ) or having received $(n=98)$ decompressive craniectomy during the acute treatment.

Measurements and Main Results: The Quality of Life after Brain Injury questionnaire was used as outcome measure with a total score from zero to 100, representing lowest and best health-related quality of life, respectively. Health-related quality of life was compared between patients with or without decompressive craniectomy for the entire cohort, for the traumatic brain injury severity (mild, moderate, severe) measured by the initial Glasgow Coma Scale, for age and time variables (age at traumatic brain injury, age at survey, elapsed time since traumatic brain injury) using the Mann-Whitney $U$ test. Differences were considered significant at a $p$ value of less than 0.05 .
Decompressive craniectomy was necessary in all initial traumatic brain injury severity groups. Eight percent more decompressive craniectomy patients reported good health-related quality of life with a Quality of Life after Brain Injury total score greater than or equal to 60 compared with the no decompressive craniectomy patients up to 10 years after traumatic brain injury $(p=0.004)$. Initially, mild classified traumatic brain injury patients had a median Quality of Life after Brain Injury total score of 83 (decompressive craniectomy) versus 62 (no decompressive craniectomy) $(p=0.028)$. Health-related quality of life regarding physical status was better in decompressive craniectomy patients $(p=0.025)$. Decompressive craniectomy showed a trend toward better health-related quality of life in the 61-85-year-old reflected by median Quality of Life after Brain Injury total scores of 62 (no decompressive craniectomy) versus 79 (decompressive craniectomy) $(p=0.06)$.

Conclusions: Our results suggest that decompressive craniectomy is associated with good health-related quality of life up to 10 years after traumatic brain injury. Thus, decompressive craniectomy may have an underestimated therapeutic potential after traumatic brain injury. (Crit Care Med 2020; 48:1157-1164)

Key Words: anxiety and depressive disorder; decompressive craniectomy; health-related quality of life; long-term outcome after traumatic brain injury; psychiatric disorders; traumatic brain injury

\footnotetext{
${ }^{1}$ Schoen Clinic Bad Aibling, Bad Aibling, Germany.

${ }^{2}$ Institute for Stroke and Dementia Research (ISD), University of Munich Medical Center, Munich, Germany.

${ }^{3}$ Department of Geriatric Psychiatry, University Hospital of Psychiatry Zurich, Zurich, Switzerland.

${ }^{4}$ Institute for Regenerative Medicine (IREM), University of Zurich, Schlieren, Switzerland.

${ }^{5}$ Institute for Medical Information Processing, Biometry, and Epidemiology (IBE), University of Munich, Munich, Germany.

${ }^{6}$ German Center for Vertigo and Balance Disorders, University of Munich Medical Center, Munich, Germany.

${ }^{7}$ Munich Cluster for Systems Neurology (Synergy), Munich, Germany.

Copyright (C) 2020 by the Society of Critical Care Medicine and Wolters Kluwer Health, Inc. All Rights Reserved.
}

DOI: 10.1097/CCM.0000000000004387
Traumatic brain injury (TBI) is the most common cause of death and disability among children and young adults and has become increasingly prevalent in the elderly $(1,2)$. For many years, experimental and clinical TBI research has primarily focused on the pathophysiology of acute and secondary brain injury, but clinical translation of therapeutic strategies has largely failed (3-7). In addition, there is evolving evidence that TBI is an ongoing chronic disease, since about one third of patients deteriorate later than 1 year after the head injury (8). Thus, chronic sequelae of TBI has moved into the focus of current research.

Decompressive craniectomy (DC) prevents secondary brain injury following experimental TBI and improves the outcome 
of a subcohort of patients with malignant ischemic stroke (6, 9). In the case of malignant ischemic stroke, DC was shown to improve long-term physical outcome and quality of life (10, 11). In contrast to stroke, two randomized controlled clinical trials evaluating physical outcome after TBI measured by the Extended Glasgow Outcome Scale (GOSE), the Randomised Evaluation of Surgery with Craniectomy for Uncontrollable Elevation of Intracranial Pressure (RESCUEicp) and Decompressive Craniectomy (DECRA) trials, failed to clearly demonstrate a beneficial effect of secondary DC in TBI patients suffering from refractory intracranial hypertension at 6 months after the brain injury $(12,13)$. However, the RESCUEicp analysis on functional outcome at 12 months showed a tendency for functional improvement indicated by higher GOSE scores. Thus, to date the effect of DC in patients suffering from TBI remains controversial. In contrast to the evaluation of DC after malignant ischemic stroke, evidence is still missing as to whether DC can improve long-term outcome after TBI and whether this improvement not only includes physical performance, but also other, more multidimensional outcome variables, such as health-related quality of life (HRQoL) (14-16). Therefore, the aim of the current study is to investigate HRQoL in patients with and without DC up to 10 years after TBI using a cross-sectional approach.

\section{PATIENTS AND METHODS}

\section{Study Design}

Four hundred thirty-nine consecutive TBI patients who were admitted to the Schoen Rehabilitation Center, Bad Aibling, Germany, between 2005 and 2015 were invited in writing to participate in this cross-sectional study. The letter contained a cover letter with a request to answer the Quality of Life after Brain Injury (QOLIBRI) questionnaire as well as an addressed and prepaid envelope. Direct telephone calls or a second contact reminder telephone call were not allowed after discharging due to the Schoen Clinic's data protection norms. In cases with severe cognitive and/or motor impairment, the patient's caregiver helped in completing the questionnaire. One major advantage of the QOLIBRI instrument is that self-completion is not a requirement and $28 \%$ of our chronic TBI patients were supported by their caregivers to complete the QOLIBRI. Patients were considered as having received decompressive craniectomy (DC+) if bone flap reimplantation was documented and not directly performed after the primary neurosurgery. Reasons for DC are described according to all available neurosurgical information in the referral letter and the medical records. All investigated TBI patients received critical care in a hospital of the Southern Upper-Bavaria Trauma Network, thereby assuring a comparable and high standard of critical and surgical care (17). According to local legislation (Bayerisches Krankenhausgesetz) and the ethical committee of the Ludwig-Maximilians University, Munich, Germany, ethics approval was not required for this study.

\section{Demographic Data}

To rule out nonresponder bias, demographic data and baseline characteristics were evaluated for the main unit $(n=439)$, the nonresponder $(n=251)$ and the QOLIBRI cohort $(n=135)$ including as follows: 1) TBI severity, 2) TBI etiology, 3) age at TBI, 4) age at survey, 5) elapsed time since TBI, 6) sex distribution, 7) whether a DC was performed, 8) whether an intracranial pressure probe or a permanent shunt device was implanted, 9) whether a tracheostomy was performed, 10) time to onset of neurorehabilitation, 11) duration of neurorehabilitation, and 12) functional status at admission, and 13) at discharge from neurorehabilitation (Table S1, Supplemental Digital Content 1, http://links.lww.com/CCM/F490). The relative frequency (\%) of each TBI severity level was quantified using the established categories: mild (Glasgow Coma Scale [GCS] 13-15), moderate (GCS 9-12), or severe (GCS 3-8), based on the initially documented score on the GCS (18).

\section{QOLIBRI: A Disease-Specific Instrument}

The QOLIBRI is validated to assess HRQoL in patients after brain injury $(19,20)$, with a QOLIBRI total score from zero to 100 , representing lowest and highest HRQoL, respectively. A QOLIBRI total score of 60 or higher represents good HRQoL; a score below 60 indicates an increased risk for one psychiatric disorder, either affective or anxiety disorder, and a score below 40 for dual psychiatric disorders (20). The QOLIBRI instrument consists of the following six scales with a total of 37 items: cognition (seven items), self (seven items), daily life and autonomy (seven items), social relationships (six items), emotions (five items), and physical problems (five items). Two major key aspects in the life scale, satisfaction and restrictions, are assessed by merging items one through four (cognition, self, daily life and autonomy, social relationships) and items five and six (emotions and physical problems), with maximum scores of 400 and 200, respectively.

\section{DC and HRQoL}

In the QOLIBRI cohort $(n=135)$, absolute frequencies of no decompressive craniectomy (DC-) and DC+ were quantified for each TBI severity group. The HRQoL, measured by the QOLIBRI total score, two key aspects, and six subscales, were compared between noncraniectomized $(n=98)$ and craniectomized ( $n=37$ ) TBI patients. Furthermore, group differences between DC- and DC+ patients and their HRQoL were analyzed for the initial classified TBI severity, the age at TBI, the age at survey, and the elapsed time since TBI.

\section{Data Management}

All QOLIBRI questionnaires were checked for completeness, and self- or caregiver-assessed rating. Each QOLIBRI responder was then assigned an interim identification number. The QOLIBRI scores were added to the demographic and basic characteristics obtained from the medical records. Then the entire data set was anonymized. 


\section{Statistical Analysis}

Descriptive statistics was used to investigate group differences between DC- $(n=98)$ and DC+ $(n=37)$ patients analyzing HRQoL measured by the QOLIBRI total score, the two key aspects and the six subscales using the Mann-Whitney $U$ test for numerical data. Distribution of HRQoL measured by the QOLIBRI total score is given as relative frequencies (\%) for DC- and DC+ patients. QOLIBRI total scores greater than or equal to 60 were descriptively compared between DC- and DC+ patients using the Fisher exact test for categorical analysis. HRQoL (QOLIBRI total score) was compared between DC- and DC+ patients for each initial classified TBI severity group, that is, mild ( $n=18)$, moderate $(n=18)$, or severe $(n=48)$ TBI using the Mann-Whitney $U$ test. To assess nonresponder bias, group differences were calculated between the QOLIBRI cohort $(n=135)$ and the nonresponder group $(n=251)$ using the Fisher exact test for categorical and the Mann-Whitney $U$ test for numerical variables. Statistical analysis was performed using R (R Core Team, Vienna, Austria). Data are reported as median with interquartile range (25-75\%) (QOLIBRI results), absolute frequency, relative frequency (\%), the mean \pm SEM, or the mean \pm sD. Differences were considered significant at $p$ value of less than 0.05 . Four of the 139 returned questionnaires were incomplete and therefore excluded.

\section{RESULTS}

\section{QOLIBRI Cohort and DC}

One-hundred thirty-five out of 439 TBI patients completed the analysis, from now on termed as the "QOLIBRI cohort."

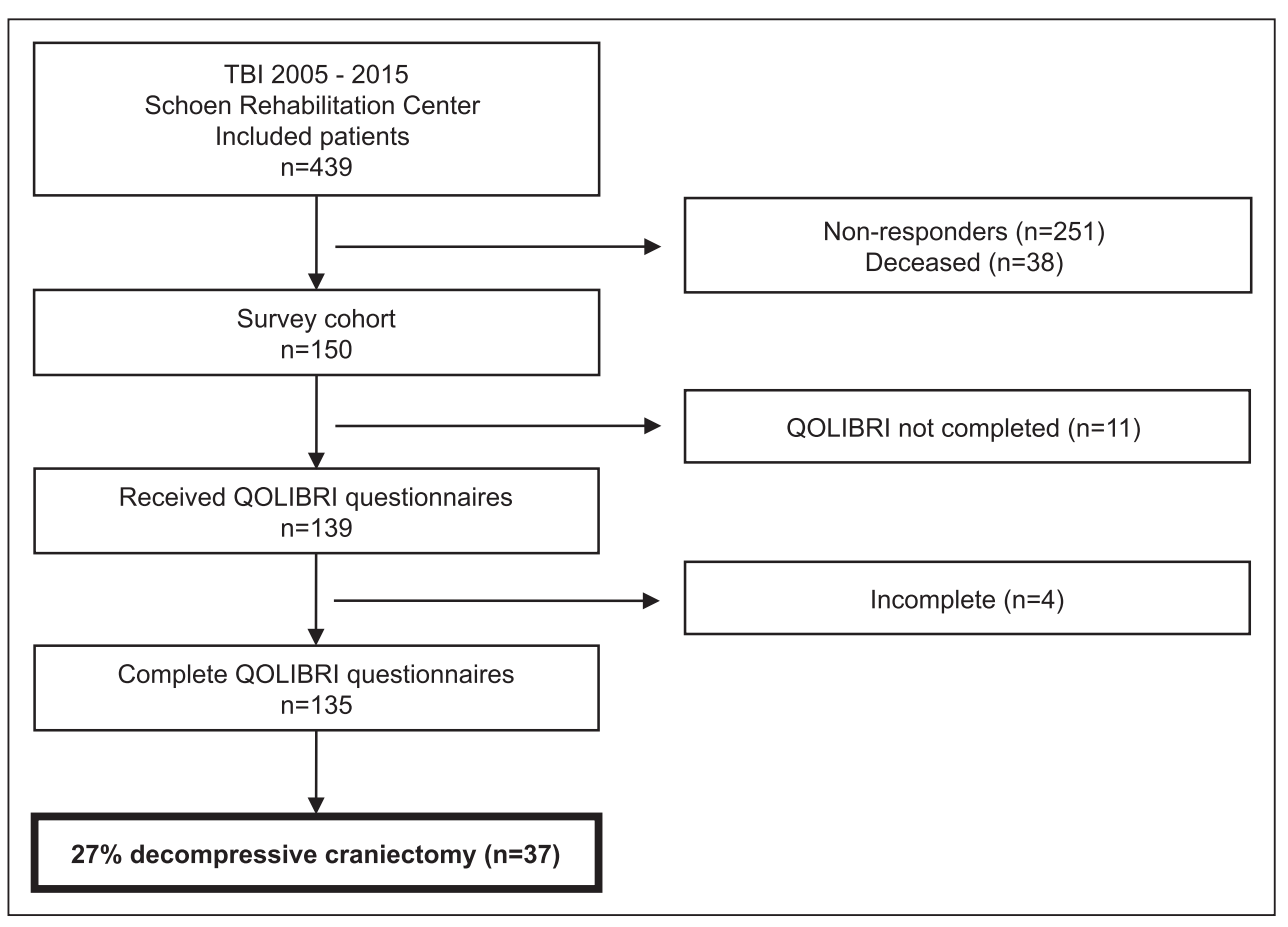

Figure 1. Flowchart depicting chronic traumatic brain injury (TBI) patients. In this cross-sectional study, 135 chronic TBI patients without $(n=98)$ or with $(n=37)$ decompressive craniectomy reported their health-related quality of life up to $10 \mathrm{yr}$ after neurorehabilitation. One-hundred thirty-five out of the 150 survey participants represent a net response rate of $90 \%$ as previously described. QOLIBRI = Quality of Life after Brain Injury.
Thirty-seven (27\%) out of 135 TBI patients underwent DC during primary care and were representative in most parameters for 133 DC+ patients of the entire cohort (Fig. 1; and Table S1, Supplemental Digital Content 1, http://links.lww.com/CCM/ F490). Patients of the QOLIBRI cohort $(n=135)$ were slightly younger when experiencing TBI $(p=0.08)$, needed shorter neurorehabilitation $(p=0.08)$ and gained better functional status at discharge from neurorehabilitation with a mean $( \pm$ sEM) modified Rankin Scale (mRS) of $2.3 \pm 0.1$ in comparison to the nonresponders $(n=251)$ with a mean $( \pm$ SEM $)$ mRS of $3.1 \pm 0.1(p$ $<0.001$ ). Thus, the nonresponders remained moderate disabled though able to walk unassisted, while patients of the QOLIBRI cohort gained good mobility and independence in activities of daily living though unable to carry out all previous activities.

\section{DC and HRQoL After TBI}

Analyzing HRQoL in DC- and DC+ patients revealed no statistical group difference. Noncraniectomized and craniectomized patients reported a median QOLIBRI total score of 67 and 75, respectively (Fig. 2A). Thus, $8 \%$ more craniectomized TBI patients reported good HRQoL with a QOLIBRI total score greater than or equal to 60 in comparison to DC- patients, namely $62 \%$ of DC- and $70 \%$ of DC+ patients $(p=0.004)$ (Fig. 2, $\boldsymbol{B}$ and $\boldsymbol{C}$ ). In detail, 23\% DC- versus 14\% DC+ were at risk for one and 15\% DC- versus 16\% DC+ for two psychiatric disorders, respectively (Fig. 2C).

\section{DC and Physical Status}

HRQoL regarding physical status was slightly better in DC+ compared with DC- patients ( $p=0.025$ ) (Fig. 3). HRQoL with respect to the two key aspects, satisfaction and restriction, as well as the other five out of six subscales did not show significant group differences (Fig. S1, Supplemental Digital Content 2, http://links.lww.com/ CCM/F542; and Table S2, Supplemental Digital Content 1, http://links.lww.com/CCM/ F490).

\section{DC and Initial TBI Severity}

DC was necessary in all initial TBI severity groups classified by the initial GCS. Six out of $18(33 \%)$ initially mild, seven out of $18(39 \%)$ moderate, and 10 out of $48(21 \%)$ severe classified TBI patients underwent DC during the acute treatment (Fig. 4). Initially mild classified TBI patients had a median QOLIBRI total score of 83 (DC+) compared with 62 

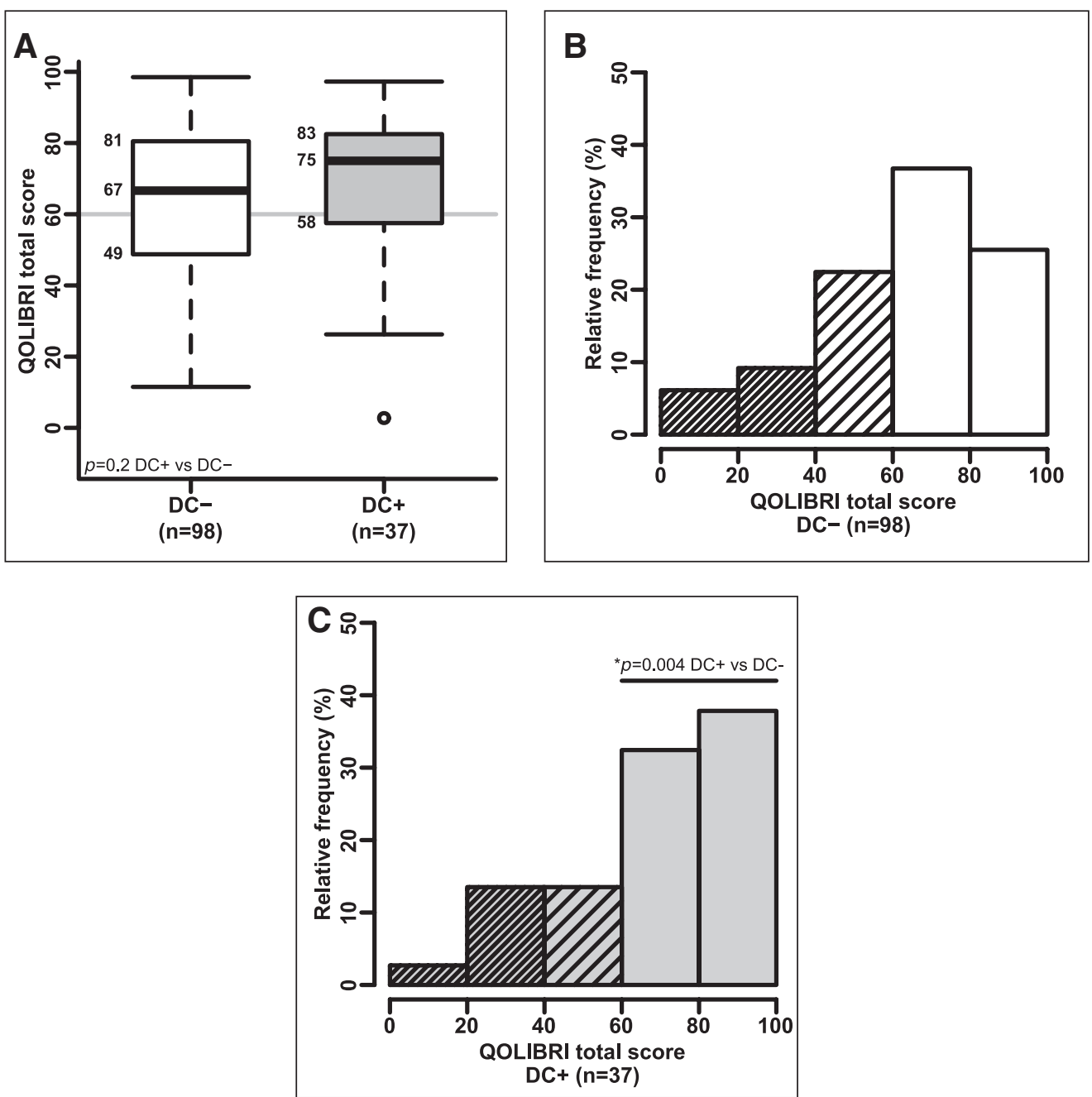

Figure 2. Decompressive craniectomy and health-related quality of life (HRQoL) following traumatic brain injury. The Quality of Life after Brain Injury (QOLIBRI) total score equal or above 60 indicates good HRQoL comparable to the healthy general population (gray line). A score below 60 (hatched) indicates a risk for one and a score below 40 (closely hatched) for both psychiatric disorders, namely anxiety or/and depressive disorder. A, Descriptive comparison of no decompressive craniectomy (DC-) (white bar) and decompressive craniectomy $(\mathrm{DC}+)$ (gray bar) patients revealed no statistical group difference using the Mann-Whitney $U$ test. B, Of DC- patients, 62\% (top, white bars). C), Of DC+ patients, 70\% (bottom, gray bars) reported good HRQoL with a QOLIBRI total score equal or above $60(p=0.004)$. Thus, $8 \%$ of patients who underwent DC were more satisfied according to their self-assessed HRQoL. The risk for one (hatched) or even two (closely hatched) psychiatric disorders differed between DC- and DC+ groups: 23\% (DC-) versus 14\% (DC+) and 15\% (DC-) versus $16 \%(D C+)$, respectively. $D C-=$ no decompressive craniectomy, $D C+=$ decompressive craniectomy.

(DC-) $(p=0.028)$ (Fig. 4). None of the DC+ and initially mild injured scored below 60, representing good HRQoL. This effect was less noticeable and not significant in moderately and not given in severely brain injured patients. Fourteen out of 51 TBI patients (28\%) received DC and were not classified for their initial TBI severity (Fig. S2, Supplemental Digital Content 2, http://links.lww.com/CCM/F542).

Reasons for DC in the initially mild TBI patients were both, that is, evacuation of mass lesion and intracranial hypertension, according to available data (Table S5, Supplemental Digital Content 1, http://links.lww.com/CCM/ F490). Most DC+ patients were initially referred to the ICU of Schoen Rehabilitation Center and suffered from moderate to severe disability according to the mRS at admission to neurorehabilitation. In detail, DC+ patients who were initially classified mild had a mean $( \pm \mathrm{sD})$ mRS of $4.7 \pm 0.5$ at admission to neurorehabilitation and the bone flap was replaced $3 \pm 1.5$ months after TBI (mean $\pm \mathrm{sD}$ ), indicating a secondary deterioration after the brain impact (Tables S4 and S5, Supplemental Digital Content 1, http:// links.lww.com/CCM/F490). DC- and DC+ groups did not differ in function at admission to neurorehabilitation but DC+ patients who were initially classified mild or moderate regained better function, while initially severe or not classified DC+ patients suffered from severe disability at discharge from neurorehabilitation (Table S4, Supplemental Digital Content 1, http://links.lww.com/ CCM/F490). DC+ patients of the initial mild to moderate TBI severity groups were able to walk unassisted or became even independent in daily activities though results were not significant in comparison to the DC-patients.

\section{DC and Age-Related Aspects for HRQoL}

DC showed a trend toward better HRQoL in the 61-85-year-old (age at TBI) reflected by a median QOLIBRI total score of $62(\mathrm{DC}-)$ versus $79(\mathrm{DC}+)(p=0.06)$ (Fig. 5). HRQoL in terms of age at survey indicate more DC+ patients with good outcome compared with the DC- patients albeit not significant (Fig. S3, Supplemental Digital Content 2, http:// links.lww.com/CCM/F542).

\section{DC and Elapsed Time Since TBI}

TBI patients, who underwent DC in the acute phase, showed a trend toward better HRQoL over time since the brain injury (Fig. S4, Supplemental Digital Content 2, http://links. lww.com/CCM/F542). During the first year after TBI, HRQoL was impaired in the noncraniectomized group with a median QOLIBRI total score of 50, while the small group of three 


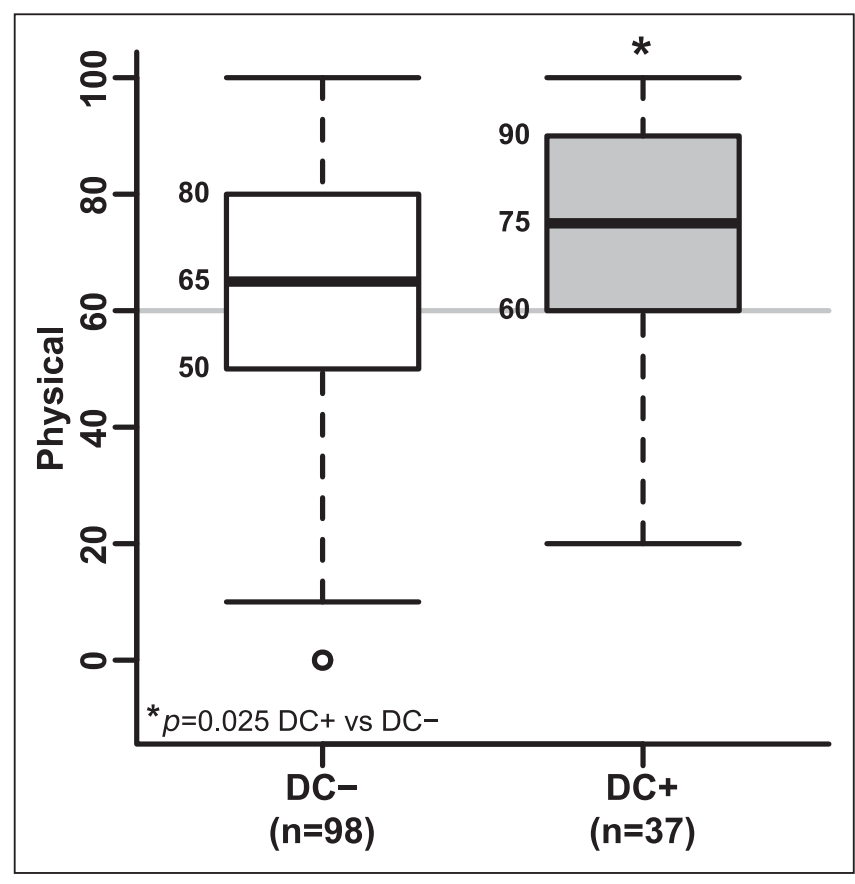

Figure 3. Decompressive craniectomy and physical status. Healthrelated quality of life regarding physical status was slightly better in $D C+$ compared with DC- patients using the Mann-Whitney $U$ test for descriptive group comparison $(p=0.025)$. DC $-=$ no decompressive craniectomy, $\mathrm{DC}+=$ decompressive craniectomy.

craniectomized TBI patients displayed a much higher, although not statistically significant, score of $97(p=0.09)$.

\section{DISCUSSION}

In this cross-sectional study, 135 craniectomized or noncraniectomized TBI patients reported on their HRQoL up to 10 years after neurorehabilitation. DC was indicated in all initially classified TBI severity groups and most beneficial in the initially mildly injured suggesting a possibly advantageous effect of DC for patients prone to secondary deterioration following TBI. A larger portion of patients with DC reported good HRQoL and were more content with their physical status. DC might result in better HRQoL outcome in the elderly, namely 61-85-year-old patients, pointing out the value of providing the highest medical care in the elderly population.

\section{Cross-Sectional Study Design and Chronic TBI Cohort}

Most demographic data and basic characteristics did not differ between the QOLIBRI cohort and the nonresponders. However, patients of the QOLIBRI cohort were slightly younger when experiencing TBI, needed shorter neurorehabilitation and gained better functional status at discharge from neurorehabilitation with a mean $\mathrm{mRS}$ of 2 , indicating slight disability with good mobility and independence in activities of daily living though unable to carry out all previous activities, while the nonresponders remained moderate disabled. Thus, a nonresponder bias cannot fully be excluded. This chronic TBI cohort experienced TBI between 2005 and 2015 and underwent neurorehabilitation at the Schoen Clinic, Bad Aibling, Germany, one of the largest neurorehabilitation centers in Europe. All patients were directly referred to neurorehabilitation by certified TBI centers, thereby receiving the highest possible standard of surgical and medical primary care. As time to onset of neurorehabilitation and neurorehabilitation procedures themselves are relevant outcome factors (21), the one center design of our study secured a high level of therapeutic homogeneity within our patient cohort.

\section{DC and Good HRQOL}

One third of patients underwent DC and 70\% of these patients reported good HRQoL as compared to only $62 \%$ in the noncraniectomized group up to 10 years after TBI. So far, only two long-term outcome studies have reported HRQoL in patients having received DC with a median follow-up of 12 months using the QOLIBRI and of 49 months using the 36-item Short Form survey instrument. Both studies did not compare HRQoL to noncraniectomized patients and included only relatively young patients with a mean age between 27 and 33 years $(22,23)$. Thus, this is the first controlled study investigating long-term HRQoL in TBI patients with or without DC.

The validity and quality of the current analysis might be further estimated by comparing our results to the already published QOLIBRI validation cohort (23). The 795 TBI patients in that cohort had a mean $( \pm \mathrm{sD})$ QOLIBRI total score of $64.6 \pm 18.2$, a value very well in line to the $64.3 \pm 22.4$ obtained in our 94 noncraniectomized control patients (Table S3, Supplemental Digital Content 1, http://links.lww.com/CCM/ F490). Hence, in comparison with a much larger TBI cohort, our HRQoL results are robust and comparable. Given the fact that there is no QOLIBRI validation cohort reporting on HRQoL following DC this current analysis might be a first step for a validation cohort after DC in TBI patients.

\section{Risk for Post-Traumatic Psychiatric Sequels}

Thirty-eight percent of noncraniectomized and 30\% of DC+ patients were at risk for psychiatric disorders following TBI, that is, DC was associated with a reduced risk for post-traumatic psychiatric disorders. This increased risk was highest in those noncraniectomized patients initially classified as mild as well as during the first year after experiencing the TBI. Most probably this increased risk, namely for anxiety and/or depressive disorders, did not preexist before injury, but was induced by TBI itself as the presented results are comparable to the QOLIBRI validation cohort, which excluded patients with preexisting psychiatric disorders (24).

\section{DC and Physical Status}

Patients who received DC during the acute phase were more satisfied with their physical status compared with DCpatients. As aforementioned, RESCUEicp failed to clearly show a better physical outcome at 6 months with a tendency for physical improvement at 12 months after TBI (12), while in the DECRA trial physical outcome was worse at 6 months 


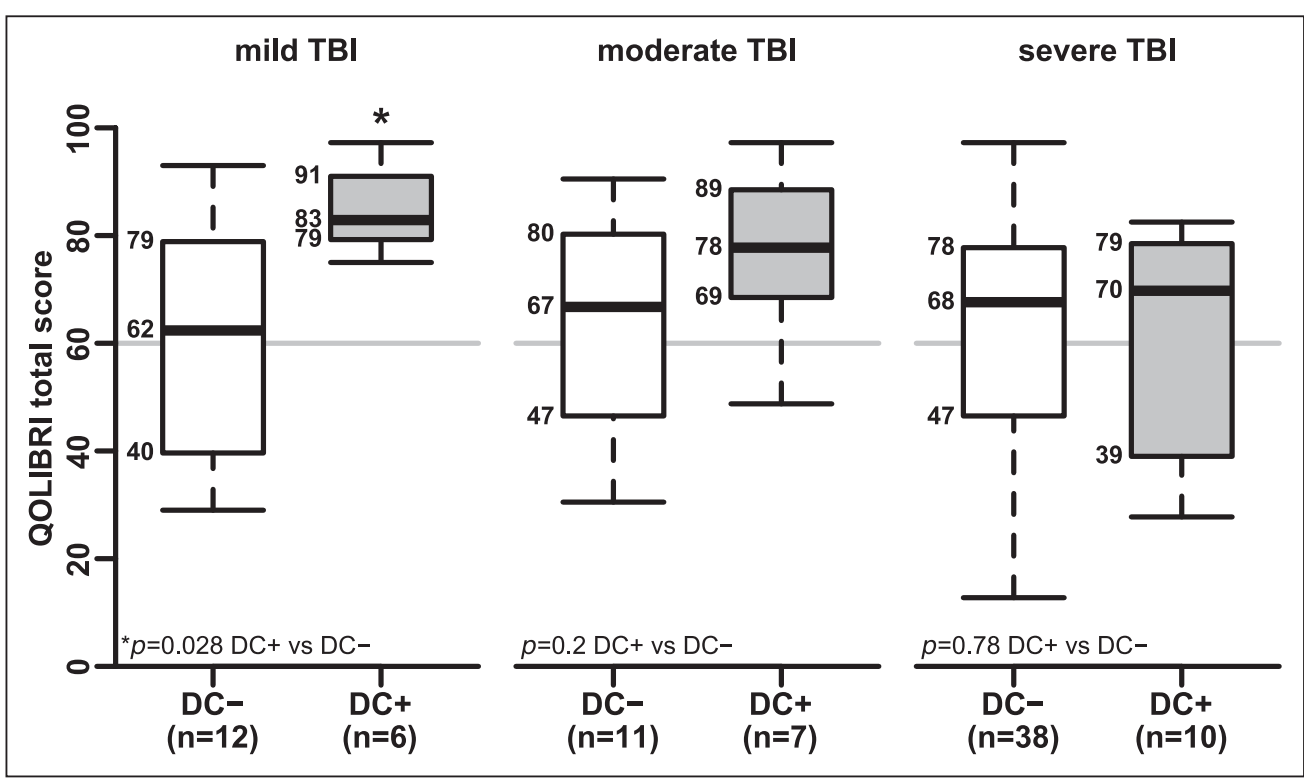

Figure 4. Decompressive craniectomy (DC) and initial traumatic brain injury (TBI) severity. DC was indicated in patients of all TBI severity groups representing the well-known occurrence of secondary deterioration. Six out of 18 initially classified as mild TBI patients suffered from moderate to severe disability when admitted to neurorehabilitation and benefitted the most from DC $(p=0.028)$. Mild, moderate, or severe TBI was defined according to the initial Glasgow Coma Scale of 13-15, 9-12, or 3-8, respectively. DC- = no decompressive craniectomy, DC+ = decompressive craniectomy, QOLIBRI = Quality of Life after Brain Injury.

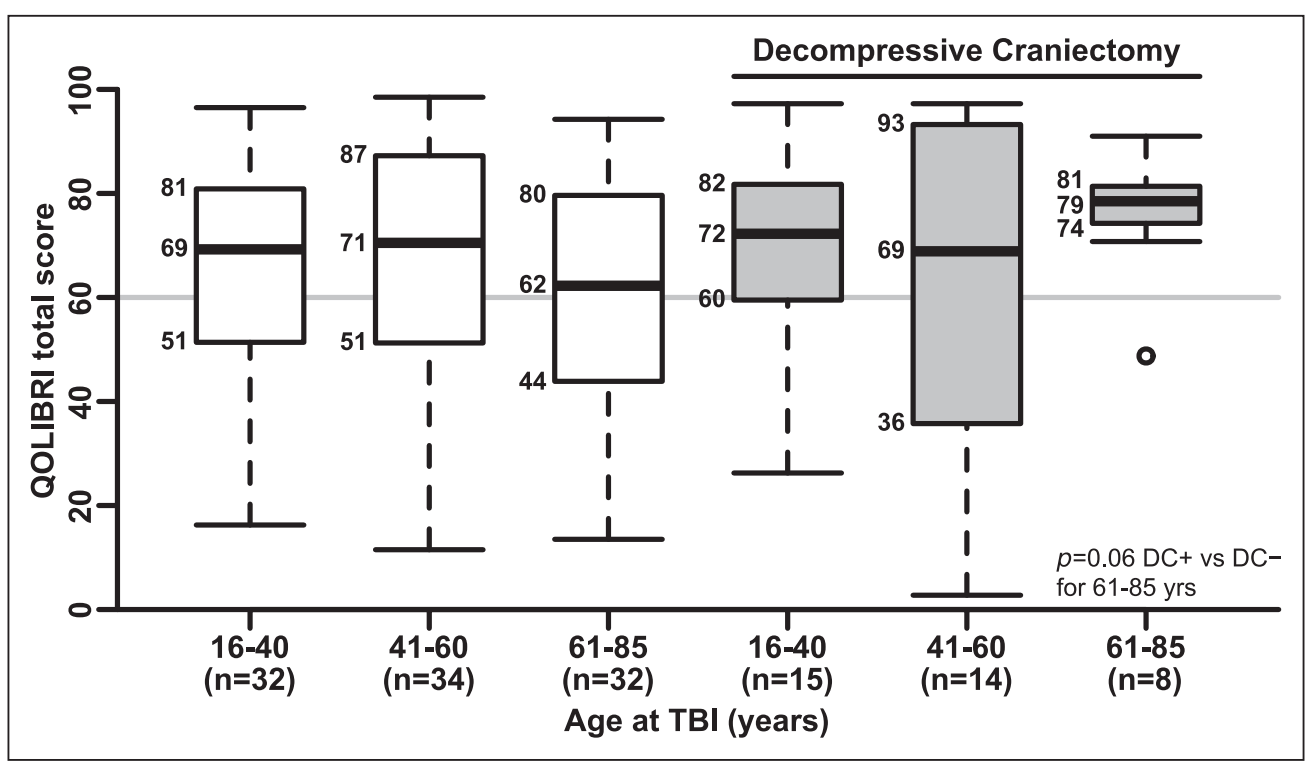

Figure 5. Decompressive craniectomy (DC) and age-related health-related quality of life (HRQoL). Age at traumatic brain injury (TBI) is not a predictor for good outcome regarding HRQoL in our chronic TBI patients. DC was associated with a better HRQoL in the 61-85-yr-old reflected by a median Quality of Life after Brain Injury (QOLIBRI) total score of $62(\mathrm{DC}-)$ versus $79(\mathrm{DC}+)$ with $p=0.06$ in the analysis for group differences. $\mathrm{DC}-=$ no decompressive craniectomy, $\mathrm{DC}+=$ decompressive craniectomy.

after DC following TBI (13). Another study reported on better functional recovery (GOSE) up to 18 months following DC after TBI in those patients with higher initial GCS (25). Thus, we suggest that objective physical disability is only one component of the overall long-term outcome after TBI and probably not the most important one in the long run. Therefore, our result underline the need for multilevel long-term outcome assessments including subjective such as HRQoL and objective measures such as the GOSE after TBI as previously suggested by Maas et al (2). Furthermore, the observation period post-TBI is particularly relevant and future studies evaluating the therapeutic efficacy of interventions and drugs will need to take this circumstance into account when investigating TBI outcome.

\section{DC in Initially Mild or Moderate Classified TBI}

In our cohort of chronic TBI patients, $27 \%$ were initially classified with mild and moderate TBIs. The fact that these patients finally needed neurorehabilitation and had comparable clinical characteristics as severe TBI patients (Table S4, Supplemental Digital Content 1, http://links.lww.com/ CCM/F490), strongly suggests that these patients secondarily deteriorated. This secondary deterioration most probably explains why $33 \%$ of initially mild (GCS 13-15) and 39\% of initially moderate (GCS 9-12) TBI patients received the indication for DC. This finding of secondary deterioration and the necessity of neurorehabilitation after TBI is well in line with a recent meta-analysis (26). Other studies even suggest that $64 \%$ to $75 \%$ of the patients initially classified with mild TBI may have an unfavorable long-term outcome ( 8 , $27,28)$. These findings underline the need for a better TBI classification beyond mild, moderate, or severe according to the GCS - as aimed by the ongoing TBI studies Transforming Research and Clinical Knowledge in Traumatic Brain Injury (TRACK-TBI) and Collaborative European NeuroTrauma Effectiveness Research in Traumatic Brain Injury (CENTER-TBI). There is no doubt, that GCS is a most valuable parameter during the emergency, but less suitable for determining TBI prognosis and outcome. Regarding HRQoL, none of the initially mild classified decompressed TBI patients scored below 60 . Thus, these patients have a very low risk to 
develop anxiety or depression and may therefore have a good long-term prognosis.

\section{DC in the Elderly and Time Since TBI}

DC was most favorable for the 61-85-year-olds with most patients reporting good HRQoL, pointing out the value of providing the highest medical care in the elderly population, even though DC is less economically attractive in the elderly and their physical outcome might be limited thereafter $(29,30)$. Interestingly, the Paris Severe Traumatic Brain Injury (PariSTBI) study analyzed HRQoL after severe TBI and showed that the QOLIBRI total score is associated with the patient's disability level. In detail, a QOLIBRI total score of equal or above 64 represents moderate recovery on the GOSE and a score equal or above 82 a good recovery on the GOSE (31). Thus, we can possibly assume moderate to good recovery in the elderly DC+ patients up to 10 years after the brain impact. During the first year after experiencing TBI, most DC- patients reported impaired HRQoL, while the majority of DC+ patients were satisfied indicating a better adaption or resilience to the post-traumatic condition in the craniectomized patients. But this latter interpretation needs to be handled with care due to the small sample size and because a selection bias during the decision process for DC cannot be excluded. If so, it is important to elucidate the patterns of decision-making for DC following TBI in the future, which will be easier due to the recently published consensus on the "Role of Decompressive Craniectomy in the Management of Traumatic Brain Injury" (32). However, previous data show that time since TBI is not a predictor for good HRQoL outcome and the majority of TBI patients already report satisfactory HRQoL 1 year after TBI.

Regarding generalizability, this is the first explorative evidence, that DC is associated with improved HRQoL up to 10 years after TBI. The only other study using the QOLIBRI questionnaire and investigating this topic, had significantly lower HRQoL values in decompressed TBI patients $(60 \pm 21$ vs $68.8 \pm 23$ in our cohort), but included only young adults treated in a tertiary care urban center with a median follow-up of 12 months (range, 6-18 mo) and a control group was missing (22). Hence, the patients' age and time of follow-up may explain the differences between the two studies, since our study showed that increased age and length of follow-up influenced HRQoL outcome.

Nevertheless, there are several limitations with this study that warrant discussion. First, we cannot fully exclude a nonresponder bias as the QOLIBRI cohort had better function at discharge from neurorehabilitation compared with the nonresponders. Second, there is a lack of detailed neurosurgical information of the treatment of DC- and DC+ patients and reasons for $\mathrm{DC}$ remain vague, that is: 1) either primary DC due to an acute bleeding or secondary neuroprotective or lifesaving, 2) the time period, management and the maximum of intracranial hypertension, 3) the exact time between brain injury and DC, and 4) the size of DC, and thus precise interpretation of the beneficial effects of DC is limited. Third, the postsurgical hemispheric diameter, a potential predictor for good functional outcome following DC, was not obtainable (33). Fourth, information on pharmacotherapy to tackle intracranial hypertension was very limited for both DC- and DC+ groups in medical reports sent to the neurorehabilitation center and certainly needs attention in future studies. Fifth, neuroimaging data using diffusion tensor imaging to precisely stratify for TBI injury patterns was not available as it was (and is still) not "state of the art" during the 2005 to 2015 study period. Sixth, TBI severity classification was limited due to missing GCS documentation in 38\% of patients-factors being well in-line with previous studies, and thus represent a common problem for TBI treatment and outcome research (26, $27,34)$. Seventh, this cross-sectional study does not provide a multilevel assessment including object measures on functional outcome, for example, the GOSE, or cognition. These assessments are in principle easy to get via phone but phoning former patients is not allowed due to the Schoen Clinic's data protection norms. However, results from the PariS-TBI study suggest that a QOLIBRI total score of equal or above 64 represents moderate recovery on the GOSE and a score equal or above 82 a good recovery on the GOSE (31). Thus, we can possibly assume moderate to good recovery in our chronic TBI cohort. Nevertheless, future and confirmatory prospective studies need to assess subjective and objective measures including HRQoL, functional and cognitive long-term outcome.

\section{CONCLUSIONS}

Our results suggest that DC is associated with good HRQoL up to 10 years after TBI. DC was indicated in all initially classified TBI severity groups with a significant better HRQoL after DC in the initially mildly injured who most probably have deteriorated, as indicated by the moderate to severe disability when admitted to neurorehabilitation. The elderly, 61-85-year-old TBI patients, showed a tendency for better HRQoL after DC. Thus, we emphasize the need for multidimensional outcome assessments to better understand the long-term outcome of TBI patients having or having not received DC during acute treatment.

\section{ACKNOWLEDGMENTS}

We are grateful to the patients, their caregivers, and their physicians for their participation in this study. We would like to especially thank Jürgen Beck for revising our article and his valuable neurosurgical comments, Benedikt Hochstrasser for technical help, and MaryJane Hill-Strathy and Jane Piper for native proof reading.

This work was performed at Schoen Clinic Bad Aibling, Kolbermoorer Strasse 72, 83043 Bad Aibling, Germany; Institute for Stroke and Dementia Research, University of Munich Medical Center, Feodor-LynenStrasse 17, 81377 Munich, Germany.

Supplemental digital content is available for this article. Direct URL citations appear in the printed text and are provided in the HTML and PDF versions of this article on the journal's website (http://journals.lww.com/ ccmjournal). 
Dr. Plesnila was supported by European Research Area Networks Network of European Funding for Neuroscience Research/Federal Ministry of Education and Research CnsAflame. The remaining authors have disclosed that they do not have any potential conflicts of interest.

Address requests for reprints to: Katrin Rauen, MD, FEBN, Department of Geriatric Psychiatry, University Hospital of Psychiatry Zurich, Minervastrasse 145, 8032 Zurich, Switzerland. E-mail: katrin.rauen@uzh.ch

\section{REFERENCES}

1. Dewan MC, Rattani A, Gupta S, et al: Estimating the global incidence of traumatic brain injury. J Neurosurg 2018 Apr 1. [online ahead of print]

2. Maas AIR, Menon DK, Adelson PD, et al; InTBIR Participants and Investigators: Traumatic brain injury: Integrated approaches to improve prevention, clinical care, and research. Lancet Neurol 2017; 16:987-1048

3. Marmarou A, Saad A, Aygok G, et al: Contribution of raised ICP and hypotension to CPP reduction in severe brain injury: Correlation to outcome. Acta Neurochir Supp/ 2005; 95:277-280

4. Mendelow AD, Teasdale GM: Pathophysiology of head injuries. $\mathrm{Br} J$ Surg 1983; 70:641-650

5. Brain Trauma Foundation: Guidelines for the Management of Severe Traumatic Brain Injury. Fourth Edition. Braintrauma.org, 2016

6. Rauen K: Experimental therapies for brain edema and raised intracranial pressure. In: Brain Edema: From Molecular Mechanisms to Clinical Practice. First Edition. Badaut J, Plesnila N, (Eds). London, Academic Press, 2017, pp 353-373

7. Loane DJ, Faden AI: Neuroprotection for traumatic brain injury: Translational challenges and emerging therapeutic strategies. Trends Pharmacol Sci 2010; 31:596-604

8. Wilson L, Stewart W, Dams-O'Connor K, et al: The chronic and evolving neurological consequences of traumatic brain injury. Lancet Neurol 2017; 16:813-825

9. Zweckberger K, Erös C, Zimmermann R, et al: Effect of early and delayed decompressive craniectomy on secondary brain damage after controlled cortical impact in mice. J Neurotrauma 2006; 23:1083-1093

10. Jüttler E, Schwab S, Schmiedek P, et al; DESTINY Study Group: Decompressive Surgery for the Treatment of Malignant Infarction of the Middle Cerebral Artery (DESTINY): A randomized, controlled trial. Stroke 2007; 38:2518-2525

11. Jüttler E, Unterberg A, Woitzik J, et al; DESTINY II Investigators: Hemicraniectomy in older patients with extensive middle-cerebralartery stroke. N Engl J Med 2014; 370:1091-1100

12. Hutchinson PJ, Kolias AG, Timofeev IS, et al; RESCUEicp Trial Collaborators: Trial of decompressive craniectomy for traumatic intracranial hypertension. N Engl J Med 2016; 375:1119-1130

13. Cooper DJ, Rosenfeld JV, Murray L, et al; DECRA Trial Investigators; Australian and New Zealand Intensive Care Society Clinical Trials Group: Decompressive craniectomy in diffuse traumatic brain injury. N Engl J Med 2011; 364:1493-1502

14. Moskowitz E, Melendez Cl, Dunn J, et al: Long-term effects of decompressive craniectomy on functional outcomes after traumatic brain injury: A multicenter study. Am Surg 2018; 84:1314-1318

15. Barthélemy EJ, Melis M, Gordon E, et al: Decompressive craniectomy for severe traumatic brain injury: A systematic review. World Neurosurg 2016; 88:411-420

16. Wettervik TS, Lenell S, Nyholm L, et al: Decompressive craniectomy in traumatic brain injury: Usage and clinical outcome in a single centre. Acta Neurochir (Wien) 2018; 160:229-237
17. Rauen K, Reichelt L, Probst $P$, et al: Quality of life up to 10 years after traumatic brain injury: A cross-sectional analysis. Health Qual Life Outcomes 2020; 18:166

18. Teasdale G, Jennett B: Assessment of coma and impaired consciousness. A practical scale. Lancet 1974; 2:81-84

19. von Steinbüchel N, Real RGL, Sasse N, et al: German validation of Quality of Life after Brain Injury (QOLIBRI) assessment and associated factors. PLoS One 2017; 12:e0176668

20. Truelle JL, Koskinen S, Hawthorne G, et al; Qolibri Task Force: Quality of life after traumatic brain injury: The clinical use of the QOLIBRI, a novel disease-specific instrument. Brain Inj 2010; 24:1272-1291

21. Konigs M, Beurskens EA, Snoep L, et al: Effects of timing and intensity of neurorehabilitation on functional outcome after traumatic brain injury: A systematic review and meta-analysis. Arch Phys Med Rehabil 2018; 99:1149-1159.e1141

22. Waqas M, Malik N, Shamim MS, et al: Quality of life among patients undergoing decompressive craniectomy for traumatic brain injury using Glasgow Outcome Scale extended and Quality of Life after Brain Injury Scale. World Neurosurg 2018; 116:e783-e790

23. Ahmadi SA, Meier U, Lemcke J: Detailed long-term outcome analysis after decompressive craniectomy for severe traumatic brain injury. Brain Inj 2010; 24:1539-1549

24. von Steinbuechel N, Covic A, Polinder S, et al: Assessment of healthrelated quality of life after TBI: Comparison of a disease-specific (QOLIBRI) with a generic (SF-36) instrument. Behav Neurol 2016; 2016:7928014

25. Ho KM, Honeybul S, Litton E: Delayed neurological recovery after decompressive craniectomy for severe nonpenetrating traumatic brain injury. Crit Care Med 2011; 39:2495-2500

26. Marincowitz C, Lecky FE, Townend W, et al: The risk of deterioration in GCS13-15 patients with traumatic brain injury identified by computed tomography imaging: A systematic review and meta-analysis. $J$ Neurotrauma 2018; 35:703-718

27. Rickels $E$, von Wild $K$, Wenzlaff $P$, et al: Schädel-Hirn-Verletzung. Germering/München, Germany, W. Zuckschwerdt Verlag, 2006, pp 149-184

28. McMillan TM, Teasdale GM, Stewart E: Disability in young people and adults after head injury: 12-14 year follow-up of a prospective cohort. J Neurol Neurosurg Psychiatry 2012; 83:1086-1091

29. Alali AS, Naimark DM, Wilson JR, et al: Economic evaluation of decompressive craniectomy versus barbiturate coma for refractory intracranial hypertension following traumatic brain injury. Crit Care Med $2014 ; 42: 2235-2243$

30. Thompson HJ, Rivara FP, Jurkovich GJ, et al: Evaluation of the effect of intensity of care on mortality after traumatic brain injury. Crit Care Med 2008; 36:282-290

31. Jourdan C, Bayen E, Vallat-Azouvi C, et al: Late functional changes post-severe traumatic brain injury are related to community reentry support: Results from the PariS-TBI cohort. J Head Trauma Rehabil 2017; 32:E26-E34

32. Hutchinson PJ, Kolias AG, Tajsic T, et al: Consensus statement from the international consensus meeting on the role of decompressive craniectomy in the management of traumatic brain injury: Consensus statement. Acta Neurochir (Wien) 2019; 161:1261-1274

33. Sauvigny T, Göttsche J, Vettorazzi E, et al: New radiologic parameters predict clinical outcome after decompressive craniectomy. World Neurosurg 2016; 88:519-525.e1

34. Ponsford J, Draper K, Schönberger M: Functional outcome 10 years after traumatic brain injury: Its relationship with demographic, injury severity, and cognitive and emotional status. J Int Neuropsychol Soc 2008; 14:233-242 